\title{
Genetic Diversity and Structure of Apomictic and Sexually Reproducing Lindera Species (Lauraceae) in Japan
}

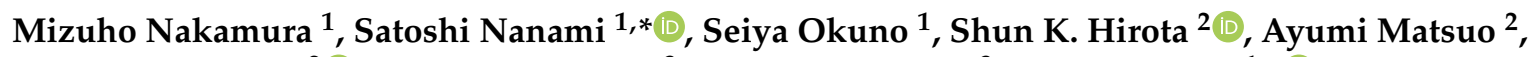 \\ Yoshihisa Suyama ${ }^{2}$ (D), Hayato Tokumoto ${ }^{3}$, Shizue Yoshihara ${ }^{3}$ and Akira Itoh ${ }^{1, *(D)}$ \\ 1 Laboratory of Plant Ecology, Graduate School of Science, Osaka City University, Osaka 558-8585, Japan; \\ m19sc015@dt.osaka-cu.ac.jp (M.N.); okuno17sc002@gmail.com (S.O.) \\ 2 Field Science Center, Graduate School of Agricultural Science, Tohoku University, Miyagi 989-6711, Japan; \\ shun.hirota@gmail.com (S.K.H.); ayumi.matsuo.b2@tohoku.ac.jp (A.M.); suyama@tohoku.ac.jp (Y.S.) \\ 3 Department of Biological Science, Osaka Prefecture University, Osaka 599-8531, Japan; \\ tokumoto@b.s.osakafu-u.ac.jp (H.T.); yoshihara@b.s.osakafu-u.ac.jp (S.Y.) \\ * Correspondence: snanami@osaka-cu.ac.jp (S.N.); itoha@osaka-cu.ac.jp (A.I.); Tel.: +81-6-6605-3167 (A.I.)
}

Citation: Nakamura, M.; Nanami, S.; Okuno, S.; Hirota, S.K.; Matsuo, A.;

Suyama, Y.; Tokumoto, H.; Yoshihara, S.; Itoh, A. Genetic Diversity and Structure of Apomictic and Sexually Reproducing Lindera Species (Lauraceae) in Japan. Forests 2021, 12, 227. https://doi.org/

10.3390/f12020227

Received: 19 December 2020

Accepted: 14 February 2021

Published: 16 February 2021

Publisher's Note: MDPI stays neutral with regard to jurisdictional claims in published maps and institutional affiliations.

Copyright: (c) 2021 by the authors. Licensee MDPI, Basel, Switzerland. This article is an open access article distributed under the terms and conditions of the Creative Commons Attribution (CC BY) license (https:// creativecommons.org/licenses/by/ $4.0 /)$.

\begin{abstract}
Research Highlights: genetic diversity in populations were compared among related shrub species with different reproductive systems. Background and Objectives: Lindera species are dioecious trees or shrubs that produce seeds by mating of males and females. To evaluate the importance of genetic diversity for the persistence of natural populations, we compared genetic information among four Lindera species in Japan. Three are dioecious shrubs (Lindera praecox, Lindera umbellata, and Lindera obtusiloba) that produce seeds by sexual reproduction. The remaining species, Lindera glauca, reproduces by apomixis; only female plants are found in Japan. Materials and Methods: all four species were sampled across a wide geographic area, from Tohoku to Kyushu, Japan. Single nucleotide polymorphisms (SNPs) were detected by multiplexed ISSR genotyping by sequencing (MIG-seq) and the resulting genetic diversity parameters were compared among populations. Results: in all sexually reproducing species, the values of observed heterozygosity were close to the expected ones and the inbreeding coefficients were nearly 0 . These results were supposed to be caused by their obligate outcrossing. The genetic difference increased, in ascending order, between a mother plant and its seeds, within populations, and across geographic space. We observed a substantial geographic component in the genetic structure of these species. For L. glauca, the genetic difference between a mother and its seeds, within populations, and across space were not significantly different from what would be expected from PCR errors. Genetic diversity within and among populations of L. glauca was extremely low. Conclusions: apomixis has the advantage of being able to found populations from a single individual, without mating, which may outweigh the disadvantages associated with the extremely low genetic diversity of L. glauca. This may explain why this species is so widely distributed in Japan. Provided that the current genotypes remain suited to environmental conditions, L. glauca may not be constrained by its limited genetic diversity.
\end{abstract}

Keywords: apomixis; clone; dioecy; geographic scale; MIG-seq; shrub; SNP

\section{Introduction}

Genetic diversity allows for environmental adaptation [1,2]. In plants, genetic diversity depends in part on the reproductive system of a given species; outcrossing species tend to be more genetically diverse [3]. Genetic diversity has been found to be higher in dioecious species, which are obligate outcrossers, compared to related cosexual species [4]. However, dioecious plants may have two reproductive disadvantages: pollination limitation because two individuals of opposite sexes are required to reproduce sexually and low colonization ability, because only half of the population contributes to seed dispersal. The seed-shadow handicap, related to reduced dispersal around female plants, typically results in a clumped 
distribution at the local scale [5], and potentially also at greater geographic scales. These advantages and disadvantages are reversed in apomictic plants.

Apomixis, the asexual formation of seeds, occurs in over 400 angiosperm genera [6]. Apomixis is considered to be advantageous for population growth and expansion, because populations can be founded by a single individual and there are no pollination limitations [7]. However, because apomictic seeds are produced without meiosis or fertilization, each seed is a genetic replica of the maternal plant unless somatic mutation occurs. Therefore, apomictic plants may have reduced disease resistance and adaptability to environmental changes.

Lindera species (Lauraceae) are dioecious trees or shrubs that produce seeds by mating of males and females. There are over 100 species of Lindera in the world, seven of which are native to Japan [8]. Of Japanese Lindera species, Lindera glauca Bl. occurs in Japan as strictly female plants that reproduce by apomixis. There are remaining questions as to how populations of this species are maintained in Japan. If genetic diversity is exceptionally low, this may support the commonly accepted theory that apomixis is advantageous in the short term due to enhanced colonization ability. However, if L. glauca has a similar level of genetic diversity to sexually reproducing species, this would suggest that the trade-off for enhanced colonization is too high, and that there is high pressure to maintain genetic diversity even in apomictic species. Previous research on Taraxacum officinale [9] and Miconia albicans [7] suggested that different genotypes were maintained in apomictic populations through somatic mutation or meiosis.

The purpose of this study was to evaluate the importance of genetic diversity for the persistence of wild plant populations. For the purpose, we compared the genetic diversity and geographic genetic structure of L. glauca to three sexually reproducing congeners, Lindera praecox $\mathrm{Bl}$., Lindera umbellata Thunb., and Lindera obtusiloba Bl., to determine the extent of variation in terms of genotype coincidence between mother plants and seeds, within populations and across geographic space.

\section{Materials and Methods}

\subsection{Focal Species}

All four species are dioecious, deciduous forest understory shrubs. Lindera praecox is endemic to Japan, while the remaining three species are found on the Asian Continent and the Japanese Archipelago. Pliocene fossils of L. praecox, L. umbellata, and L. obtusiloba have been found in Japan [10], and Pleistocene fossils of L. glauca have been found [11]. The species flower in spring, from late February to early May, with each species flowering for 2-4 weeks [8,12]. The flowers, which are borne in pseudo-umbel-shaped inflorescences, are small (4-9 $\mathrm{mm}$ in diameter), dish-shaped, yellow or green in color, and unisexual, with sterile rudiments of the other sex [12].

In the past, the seeds of L. praecox were used to press oil for lanterns [13]. Lindera umbellata extracts had the effects of prevention of onset and progression of nephropathy [14]. Lindera obtusiloba has been widely used in a traditional medicine as the anti-allergic inflammatory effect [15]. The fruits of L. glauca contain some aromatic essential oils, alkaloids, terpenoids, and flavonoids, which are widely employed in the essence, medicine, and chemical industries [16].

The sex ratio of populations of L. praecox, L. umbellata, and L. obtusiloba is approximately 1:1 in forests in western Japan [12]. Both male and female flowers attract insect pollinators, primarily Diptera and Coleoptera [8]. Regarding L. glauca, only female plants are found in Japan, but both males and females are found on the Asian Continent [17,18]. The sex ratio of L. glauca in the wild in mainland China is estimated to be predominantly female [19]. Dupont [17] conducted bagging experiments to clarify whether or not pollination was necessary for seed set of Lindera species. She covered flower buds on branches of female plants of L. umbellata, L. obtusiloba, and L. glauca with nylon meshes to keep pollen out. The results for L. umbellata and L. obtusiloba showed that pollination was essential for their seed set. On the other hand, females of L. glauca produced seeds without pollination 
or male flowers, indicating apomixis. For some woody plants, clonal growth arises by wide-ranging roots that produce new shoots, termed stolon. However, the four Lindera species do not reproduce laterally by stolons. Hence, stems derived from an individual can easily identified.

\subsection{Field Collections and DNA Extraction}

We collected leaves from 105 individuals of L. glauca in natural vegetations at 29 locations, representing the entirety of its distribution in Japan (Supplementary Material Table S1). We tried to collect up to five individuals from one location, but in some locations, we could only find one. As a result, leaves were taken from one to five individuals per location. From six locations among the 29 locations, i.e., Takaragaike, Kasugayama, Myokensan, Shigisan, Okayama-shi, and Umamiyama, five seeds from each selected mature individual were taken (Supplementary Material Table S2). For L. praecox, we collected leaves from 45 individuals (one to five individuals per location) in natural vegetations at 11 locations (Table S1). From two locations, among the 11 locations, i.e., Mitarai Valley and Tsuno, six to twelve seeds were collected from each of six mature individuals (Table S2). For L. umbellata, we collected leaves from 27 individuals at six locations on Honshu Island (two-five individuals per location) (Table S1). From a mature individual among six locations, i.e., Mitarai Valley, five seeds were collected (Table S2). For L. obtusiloba, we collected leaves from 39 individuals at eight locations on Honshu, Shikoku, and Tsushima Islands (four-five individuals per location) (Table S1). From two individuals at Mitarai Valley, 11 and 12 seeds were collected (Table S2). We recorded the geographic location, i.e., latitude and longitude, and diameter at breast height (DBH; $1.3 \mathrm{~m})$ of each sampled individual (Table S1).

DNA was extracted from leaves and seed cotyledons for each species using a DNA extraction kit (DNeasy Plant Mini Kit; QIAGEN, Tokyo, Japan) or a DNA extraction buffer (UniversAll $^{\mathrm{TM}}$ Extraction Buffer II; Yeastern Biotech, New Taipei City, Taiwan).

\subsection{Multiplexed ISSR Genotyping by Sequencing (MIG-Seq) Analysis}

DNA sequences were subjected to MIG-seq analysis [20] and single nucleotide polymorphisms (SNPs) were identified. In MIG-seq analysis, the region between single sequence repeats (SSRs), i.e., the inter-SSR (ISSR), is amplified by polymerase chain reaction (PCR). Approximately $1.5 \mathrm{ng} / \mu \mathrm{L}$ of DNA was used for the first PCR, as template DNA. The concentration of the DNA solution was measured using a Quantus ${ }^{\mathrm{TM}}$ Fluorometer (Promega, Madison, WI, USA), and the concentration of the template DNA solution was adjusted by adding sterile water.

For the first PCR, a mixture of primers was prepared from eight types of forward and reverse primers intended to amplify the ISSR region. The fragments were amplified with a Multiplex PCR Assay Kit Ver. 2 (TaKaRa Bio Inc., Shiga, Japan) using $7 \mu \mathrm{L}$ reaction volumes containing $1.0 \mu \mathrm{L}$ of template DNA, $1.4 \mu \mathrm{L}$ of PCR primers (mix of primers, each at a concentration of $2.0 \mu \mathrm{M}$ ), $3.5 \mu \mathrm{L}$ of $2 \times$ Multiplex PCR Buffer, $0.035 \mu \mathrm{L}$ of Multiplex PCR Enzyme Mix and $1.065 \mu \mathrm{L}$ of water. The PCR cycling conditions used for the Verti 96-Well Thermal Cycler (Applied Biosystems, Foster City, CA, USA) were as follows: $94{ }^{\circ} \mathrm{C}$ for $1 \mathrm{~min}$ followed by 27 cycles of $94{ }^{\circ} \mathrm{C}$ for $30 \mathrm{~s}, 38^{\circ} \mathrm{C}$ for $1 \mathrm{~min}, 72{ }^{\circ} \mathrm{C}$ for $1 \mathrm{~min}$, and finally $72{ }^{\circ} \mathrm{C}$ for $10 \mathrm{~min}$.

The second PCR used the products of the first PCR diluted to a concentration of 1:50 by adding sterile water. The second PCR was performed to add complementary sequences as an index to both ends of the first PCR products to identify individuals. The fragments were amplified with PrimeSTAR GXL DNA polymerase (TaKaRa Bio Inc., Shiga, Japan) using $12 \mu \mathrm{L}$ reaction volumes containing $2.5 \mu \mathrm{L}$ of diluted first PCR products as template DNA, $2.4 \mu \mathrm{L}$ of second PCR primers (mix of primers, each at a concentration of $2.0 \mu \mathrm{M}$ ), $2.4 \mu \mathrm{L}$ of $5 \times$ PrimeSTAR Buffer, $0.96 \mu \mathrm{L}$ of dNTP mixture, $0.24 \mu \mathrm{L}$ of PrimeSTAR GXL polymerase, and $3.5 \mu \mathrm{L}$ of water. The PCR cycling conditions were as follows: 12 cycles of $98{ }^{\circ} \mathrm{C}$ for $10 \mathrm{~s}, 54{ }^{\circ} \mathrm{C}$ for $15 \mathrm{~s}$, and $68{ }^{\circ} \mathrm{C}$ for $1 \mathrm{~min}$. After purification of the second 
PCR products with AMPure XP magnetic beads, only 300-800 bp fragments with a size suitable for sequencing were selected by the Blue Pippin DNA Size Selection System (Sage Science, Beverly, MA, USA). Tape Station 4200 (Agilent Technologies, Santa Clara, CA, USA) and Qubit fluorometer (Life Technology, Carlsbad, CA, USA) were used to measure the frequency distribution of fragment lengths and the amount of fragments. Prepared to a concentration of $12 \mathrm{pM}$, the DNA library was used for sequencing on an Illumina MiSeq sequencing platform using a MiSeq Reagent Kit v3 (Illumina, San Diego, CA, USA). The sequencing results were input into Stacks v.1.47 [21] to identify loci and detect SNPs. Following Catchen et al. [22], we used "ustacks" for building loci, "cstacks" for creating the loci catalogue for all samples, and "sstacks" for checking against the catalog. Finally, the "population" module was used to estimate a "population genetics statistic" from a population of individual samples.

\subsection{Data Analyses}

Only loci with more than $50 \%$ of the population and less than $60 \%$ heterozygosity were extracted. For each species, the number of alleles $(\mathrm{Na})$, number of effective alleles $(\mathrm{Ne})$, observed heterozygosity $(\mathrm{Ho})$, expected heterozygosity $(\mathrm{He})$, and an inbreeding coefficient $\left(F_{\text {IS }}\right)$ were estimated using GenAlEx v.6.5 [23]. Genotypes, including those from seed samples, were compared to identify clones and to assess genotype diversity for each species.

We calculated the proportion of different alleles on each SNP loci as follows: if individuals shared an identical genotype (e.g., $\mathrm{A} / \mathrm{T}$ and $\mathrm{A} / \mathrm{T}$ ), the proportion of different alleles was 0 ; if one allele differed between individuals (e.g., A/T and A/G), it was 0.5; and if the genotypes differed completely (e.g., A/T and G/C), it was 1 . Once all loci had been assessed, the mean proportion of different alleles was defined as the genetic difference between individuals. To investigate the extent to which genetic variation occurs in apomictic seed production relative to sexual reproduction, the genetic difference between mother plants and their seeds was determined. We then determined the genetic difference between grown-up individuals to assess genotype diversity within a population by pairings limited to individuals growing in the same location. For the assessment the genotype diversity across the entire study area, the genetic difference was calculated for all pairs of grown-up individuals after combining all individuals sampled from all locations into a single pool. We estimated the possibility that pseudo-variant loci occurred originating from PCR errors using the genotype data from pairs of replicates of the same individual. We performed PCR amplification and genotyping twice for seven individuals of L. glauca and six individuals of L. praecox, L. umbellata, and L. obtusiloba. We defined the pseudo-variant rate as the genetic difference between replicates of the same individual.

To examine the distribution of genetic variation within and among studied locations and to explore genetic relationships among individuals and geographic genetic structure, pairwise individual-by-individual genetic distance matrixes were calculated for codominant data [24] using GenAlEx v.6.5. To deal with missing data, we selected the option "interpolate missing". Based on the matrixes, we conducted analysis of molecular variance (AMOVA) using the R package "pegas" v.0.14 [25] and a principal coordinate analysis ( $\mathrm{PCOA})$ to understand the genetic relationships among individuals and among locations in detail using the R package "vegan" v.2.5 [26]. To evaluate the relationship between geographic distance and genetic divergence, we calculated a fixation index $\left(F_{\mathrm{ST}}\right)$ value between populations, and assessed the correlation between $F_{\mathrm{ST}}$ and the geographic distance between populations using the R package "ape" v.5.4 [27].

\section{Results}

\subsection{Genetic Diversity Parameters}

For L. glauca, we obtained 246 SNP loci from 147 samples, including 105 individuals, replicates of seven individuals and 35 seeds, taken from 29 populations. The mean $\mathrm{Na}$ and Ne were 0.903 and 0.894 , respectively. He varied from 0.000 to 0.018 , whereas Ho varied 
from 0.000 to 0.027 . F IS was negative excluding Hagi $(\mathrm{HG})$ (range: -0.760 to 0.429 ; mean $=$ -0.441) (Table 1).

Table 1. Genetic diversity parameters of the 29 populations of Lindera glauca (Lauraceae) in Japan based on 246 single nucleotide polymorphism (SNP) loci. Letters in parentheses are abbreviations of sampling location.

\begin{tabular}{ccccccc}
\hline Sampling Location & $N$ & Na & Ne & He & Ho & $F_{\text {IS }}$ \\
\hline Sendai, Miyagi (SD) & 1 & 0.687 & 0.687 & 0.004 & 0.008 & - \\
Hachioji, Tokyo (HT) & 2 & 0.796 & 0.792 & 0.012 & 0.021 & -0.760 \\
Sagamihara, Kanagawa (SH) & 2 & 0.936 & 0.927 & 0.012 & 0.019 & -0.538 \\
Kawasaki, Kanagawa (KW) & 3 & 0.909 & 0.896 & 0.01 & 0.014 & -0.401 \\
Nagano, Nagano (NG) & 5 & 1.023 & 1.005 & 0.014 & 0.021 & -0.504 \\
Kasugai, Aichi (I) & 5 & 1.023 & 1.009 & 0.012 & 0.018 & -0.461 \\
Aioiyama, Aichi (A) & 5 & 1.034 & 1.021 & 0.014 & 0.02 & -0.381 \\
Hino, Shiga (HN) & 3 & 0.002 & 0.003 & 0.01 & 0.018 & -0.749 \\
Takaragaike, Kyoto (T) & 5 & 1.023 & 1.017 & 0.013 & 0.018 & -0.328 \\
Kasugayama, Nara (K) & 5 & 1.019 & 1.008 & 0.011 & 0.018 & -0.598 \\
Myokensan, Osaka (M) & 5 & 1.038 & 1.029 & 0.018 & 0.027 & -0.499 \\
Shigisan, Osaka (S) & 5 & 1.026 & 1.020 & 0.011 & 0.016 & -0.434 \\
Takedao, Hyogo (TK) & 4 & 0.996 & 0.981 & 0.014 & 0.02 & -0.496 \\
Okayamashi, Okayama (O) & 5 & 1.000 & 0.995 & 0.008 & 0.012 & -0.578 \\
Hiroshimashi, Hiroshima (H) & 4 & 0.909 & 0.900 & 0.01 & 0.016 & -0.665 \\
Masuda, Shimane (MS) & 5 & 1.038 & 1.022 & 0.016 & 0.019 & -0.222 \\
Tsuwano, Shimane (TW) & 2 & 0.977 & 0.972 & 0.006 & 0.009 & -0.527 \\
Izumo, Shimane (IZ) & 5 & 1.038 & 1.023 & 0.013 & 0.017 & -0.263 \\
Yamaguchishi, Yamaguchi (YG) & 5 & 1.023 & 1.012 & 0.007 & 0.01 & -0.360 \\
Hagi, Yamaguchi (HG) & 2 & 0.962 & 0.961 & 0.003 & 0.002 & 0.429 \\
Bizan, Tokushima (B) & 3 & 1.019 & 1.015 & 0.012 & 0.019 & -0.565 \\
Iinoyama, Kagawa (IN) & 5 & 0.985 & 0.961 & 0.014 & 0.019 & -0.391 \\
Shiunzan, Kagawa (SU) & 5 & 1.049 & 1.021 & 0.018 & 0.024 & -0.306 \\
Tsuno, Kochi (TN) & 3 & 0.887 & 0.881 & 0.009 & 0.015 & -0.618 \\
Sameura, Kochi (SM) & 5 & 1.038 & 1.018 & 0.011 & 0.016 & -0.377 \\
Tsushima, Nagasaki (TS) & 1 & 0.596 & 0.596 & 0.008 & 0.015 & - \\
Umamiyama, Fukuoka (UM) & 3 & 0.898 & 0.886 & 0.011 & 0.016 & -0.435 \\
Sasga, Saga (SA) & 1 & 0.811 & 0.811 & 0.008 & 0.015 & - \\
Sannotake, Kumamoto (SN) & 1 & 0.457 & 0.457 & 0 & 0 & - \\
Regional mean & & 0.903 & 0.894 & 0.011 & 0.016 & -0.441 \\
\hline
\end{tabular}

$\mathrm{N}$, number of individuals; $\mathrm{Na}$, number of observed alleles; $\mathrm{Ne}$, effective number of alleles; $\mathrm{He}$, expected heterozygosity; Ho, observed heterozygosity; $F_{\mathrm{IS}}$ : inbreeding coefficient.

For L. praecox, we obtained 355 SNP loci from 114 samples, including 45 individuals, replicates of six individuals and 63 seeds, taken from 11 populations. The mean $\mathrm{Na}$ and $\mathrm{Ne}$ were 1.025 and 0.968 , respectively. He ranged from 0.008 to 0.075 , whereas Ho ranged from 0.016 to 0.070 . $F_{\text {IS }}$ was positive in all populations excluding Hiroshima (H) (range: -0.223 to 0.151 ; mean $=0.057$ ) (Table 2).

For L. umbellata, we obtained 280 SNP loci from 38 samples, including 27 individuals, replicates of six individuals and five seeds, taken from six populations. The mean $\mathrm{Na}$ and Ne were 1.210 and 1.115, respectively. He ranged from 0.056 to 0.096 , whereas Ho ranged from 0.075 to 0.114 . F IS was negative in all populations excluding Hino (HN) and Myokensan (M) (range: -0.356 to $0.020 ;$ mean $=-0.142)($ Table 3$)$. 
Table 2. Genetic diversity parameters of the 11 populations of Lindera praecox (Lauraceae) in Japan based on 355 SNP loci. Letters in parentheses are abbreviations of sampling location.

\begin{tabular}{ccccccc}
\hline Sampling Location & $N$ & Na & Ne & He & Ho & $F_{\text {IS }}$ \\
\hline Sendai, Miyagi (SD) & 1 & 0.402 & 0.402 & 0.008 & 0.016 & - \\
Tsukubasan, Ibaraki (TB) & 5 & 1.136 & 1.064 & 0.063 & 0.056 & 0.108 \\
Hachioji, Tokyo (HT) & 1 & 0.786 & 0.786 & 0.022 & 0.043 & - \\
Nagano, Nagano (NG) & 5 & 1.125 & 1.059 & 0.059 & 0.054 & 0.094 \\
Hino, Shiga (HN) & 5 & 1.146 & 1.073 & 0.070 & 0.070 & 0.010 \\
Mitarai, Nara (MT) & 5 & 1.164 & 1.083 & 0.061 & 0.057 & 0.072 \\
Kongosan, Osaka (KG) & 5 & 1.195 & 1.114 & 0.075 & 0.068 & 0.094 \\
Takedao, Hyogo (TK) & 5 & 1.160 & 1.072 & 0.062 & 0.052 & 0.151 \\
Hiroshimashi, Hiroshima (H) & 3 & 0.996 & 0.960 & 0.043 & 0.052 & -0.223 \\
Tsuno, Kochi (TN) & 5 & 1.094 & 1.031 & 0.055 & 0.047 & 0.140 \\
Umamiyama, Fukuoka (UM) & 5 & 1.070 & 1.009 & 0.054 & 0.050 & 0.071 \\
Regional mean & & 1.025 & 0.968 & 0.052 & 0.051 & 0.057
\end{tabular}

$\mathrm{N}$, number of individuals; $\mathrm{Na}$, number of observed alleles; $\mathrm{Ne}$, effective number of alleles; $\mathrm{He}$, expected heterozygosity; $H \mathrm{o}$, observed heterozygosity; $F_{\mathrm{IS}}$ : inbreeding coefficient.

Table 3. Genetic diversity parameters of the six populations of Lindera umbellata (Lauraceae) in Japan based on 280 SNP loci. Letters in parentheses are abbreviations of sampling location.

\begin{tabular}{ccccccc}
\hline Sampling Location & $\boldsymbol{N}$ & $\mathbf{N a}$ & $\mathbf{N e}$ & $\mathbf{H e}$ & $\mathbf{H o}$ & $\boldsymbol{F}_{\text {IS }}$ \\
\hline Sendai, Miyagi (SD) & 5 & 1.120 & 1.102 & 0.071 & 0.089 & -0.252 \\
Tsukubasan, Ibaraki (TB) & 5 & 1.234 & 1.142 & 0.096 & 0.114 & -0.183 \\
Hino, Shiga (HN) & 5 & 1.268 & 1.133 & 0.090 & 0.088 & 0.020 \\
Mitarai, Nara (MT) & 5 & 1.204 & 1.109 & 0.080 & 0.087 & -0.089 \\
Myokensan, Osaka (M) & 5 & 1.257 & 1.138 & 0.084 & 0.084 & 0.006 \\
Takedao, Hyogo (TK) & 2 & 1.102 & 1.069 & 0.056 & 0.075 & -0.356 \\
Regional mean & & 1.210 & 1.115 & 0.079 & 0.089 & -0.142 \\
\hline
\end{tabular}

$\mathrm{N}$, number of individuals; $\mathrm{Na}$, number of observed alleles; $\mathrm{Ne}$, effective number of alleles; $\mathrm{He}$, expected heterozygosity; Ho, observed heterozygosity; $F_{\mathrm{IS}}$ : inbreeding coefficient.

For L. obtusiloba, 688 SNP loci were obtained from 68 samples, including 39 individuals, replicates of six individuals and 23 seeds, taken from eight populations. The mean $\mathrm{Na}$ and Ne were 1.227 and 1.124, respectively. He ranged from 0.071 to 0.097 , whereas Ho ranged from 0.073 to 0.084 . $F_{\text {IS }}$ was close to zero in all population (range: -0.087 to 0.149 ; mean $=$ 0.022) (Table 4).

Table 4. Genetic diversity parameters of the eight populations of Lindera obtusiloba (Lauraceae) in Japan based on 688 SNP loci. Letters in parentheses are abbreviations of sampling location.

\begin{tabular}{ccccccc}
\hline Sampling Location & $N$ & Na & Ne & He & Ho & $\boldsymbol{F}_{\text {IS }}$ \\
\hline Nagano, Nagano (NG) & 5 & 1.268 & 1.14 & 0.097 & 0.083 & 0.149 \\
Hino, Shiga (HN) & 5 & 1.233 & 1.133 & 0.085 & 0.083 & 0.027 \\
Mitarai, Nara (MT) & 5 & 1.203 & 1.108 & 0.078 & 0.084 & -0.078 \\
Myokensan, Osaka (M) & 5 & 1.248 & 1.134 & 0.084 & 0.073 & 0.133 \\
Kongosan, Osaka (KG) & 5 & 1.264 & 1.145 & 0.093 & 0.084 & 0.095 \\
Takedao, Hyogo (TK) & 5 & 1.249 & 1.138 & 0.085 & 0.084 & 0.008 \\
Iinoyama, Kagawa (IN) & 4 & 1.177 & 1.103 & 0.072 & 0.079 & -0.087 \\
Tsushima, Nagasaki (TS) & 5 & 1.172 & 1.095 & 0.071 & 0.076 & -0.070 \\
Regional mean & & 1.227 & 1.124 & 0.083 & 0.081 & 0.022 \\
\hline
\end{tabular}

$\mathrm{N}$, number of individuals; $\mathrm{Na}$, number of observed alleles; $\mathrm{Ne}$, effective number of alleles; $\mathrm{He}$, expected heterozygosity; Ho, observed heterozygosity; $F_{\mathrm{IS}}$ : inbreeding coefficient.

\subsection{Clone Identification and Genotype Diversity}

For L. praecox, L. umbellata, and L. obtusiloba, pseudo-variant rates ranged from 0.005 to $0.013,0.005-0.012$, and $0.004-0.070$, respectively (Figure 1a-c). Pseudo-variant rates in the seven pairs of replicated L. glauca samples ranged from 0.004 to 0.017 (Figure 1d). 


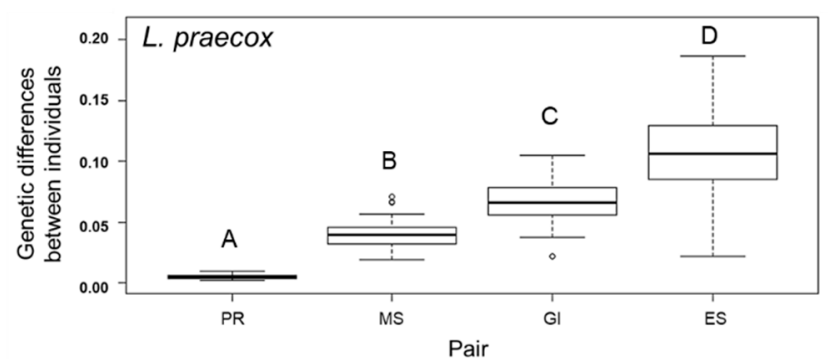

(a)

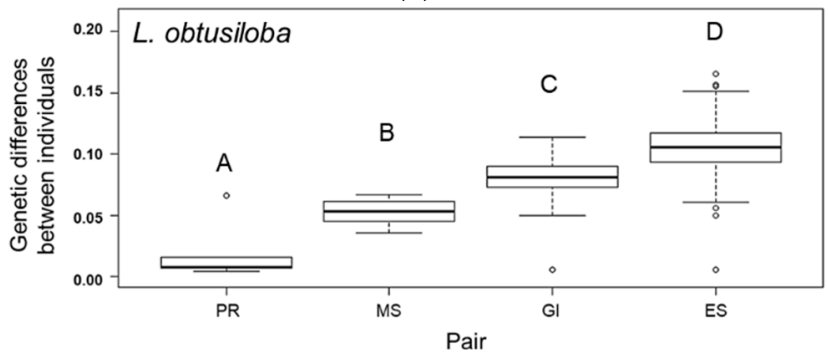

(c)

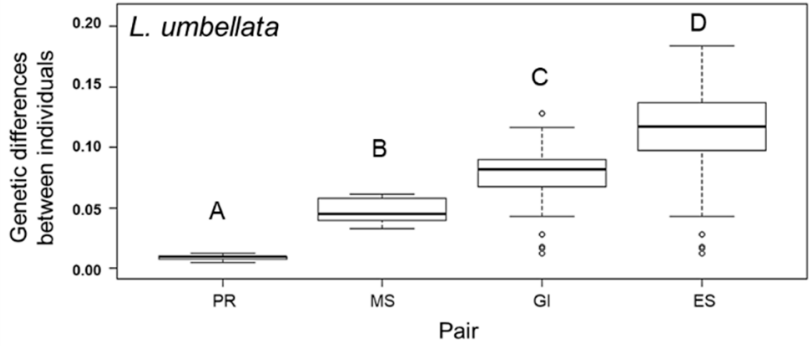

(b)

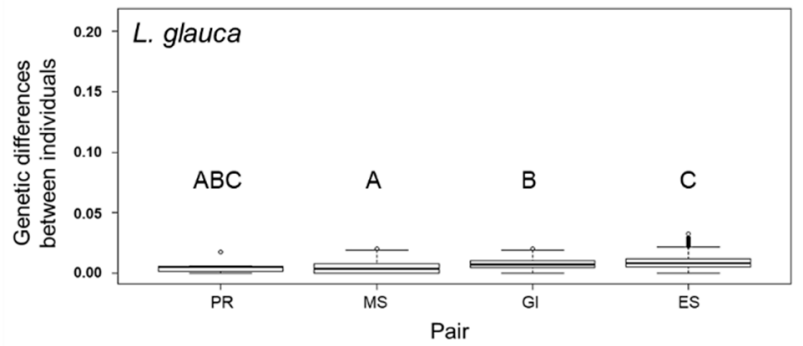

(d)

Figure 1. Boxplot of the genetic difference between individuals of (a) Lindera praecox, (b) L. umbellata, (c) L. obtusiloba, and (d) L. glauca (Lauraceae). The box represents the interquartile range (25th-75th percentiles); while the line across the box indicates the median. Whiskers extend to the 1.5-fold of the interquartile range. The circles indicate outliners. Values denoted by the same letters are not significantly different from each other at $p<0.05$ (Kruskal-Wallis test and Wilcoxon test with sequential Bonferroni correction). PR; the possibility that pseudo-variant loci occurred originating from PCR errors, MS; the genetic difference between mother plants and their seeds, GI; the genetic difference between grown-up individuals by parings individuals growing in the same location, and ES; the genetic difference calculated for all pairs of grown-up individuals after combining all individuals sampled from all locations into a single pool.

For L. praecox, L. umbellata, and L. obtusiloba, the genetic difference between a mother plant and its seeds, the genetic difference within populations, and the genetic difference across the entire study area were significantly greater than would be expected from pseudovariant rate $(p<0.05$; Kruskal-Wallis test and Wilcoxon test with sequential Bonferroni correction; Figure 1). Additionally, the genetic differences were significantly different among these three scales $(p<0.05$; Wilcoxon test with sequential Bonferroni correction; Figure $1 \mathrm{a}-\mathrm{c}$ ). For L. glauca, the genetic difference at these three scales was not significantly different than what would be expected from pseudo-variant rates ( $p>0.05$, KruskalWallis test and Wilcoxon test with sequential Bonferroni correction; Figure 1d). However, the genetic difference among the three scales was significantly different from each other $(p<0.05$; Wilcoxon test with sequential Bonferroni correction; Figure 1d).

\subsection{Genetic Structure}

Results of AMOVA indicated that genetic variation within populations was greater than among populations for L. praecox, at 56\% and 44\%, respectively (Table 5). For L. umbellata, genetic variation was greater within populations (53\%), followed by among population (47\%). Genetic variation among populations of L. obtusiloba $(76 \%)$ was substantially larger than within populations (24\%). For L. glauca, geographic differences contributed to only $9 \%$ of the genetic variation within this species. 
Table 5. Analysis of Molecular Variance (AMOVA) results for the studied four Lindera populations.

\begin{tabular}{|c|c|c|c|c|c|}
\hline \multicolumn{2}{|r|}{ Source of Variation } & \multirow[t]{2}{*}{$d f$} & \multirow[t]{2}{*}{ Sum of Squares } & \multirow[t]{2}{*}{ Variance Components } & \multirow[t]{2}{*}{$\%$} \\
\hline (A) & L. praecox & & & & \\
\hline & Among population & 10 & $427,635.500$ & 8034.000 & 44 \\
\hline & Within population & 34 & $352,842.900$ & $10,378.000$ & 56 \\
\hline & Total & 44 & $780,478.400$ & $18,412.000$ & \\
\hline \multirow[t]{4}{*}{ (B) } & L. umbellata & & & & \\
\hline & Among population & 5 & $57,977.820$ & 2084.700 & 47 \\
\hline & Within population & 21 & $48,930.350$ & 2330.000 & 53 \\
\hline & Total & 26 & $106,908.170$ & 4414.700 & \\
\hline \multirow[t]{4}{*}{ (C) } & L. obtusiloba & & & & \\
\hline & Among population & 7 & $477,950.100$ & 9319.100 & 76 \\
\hline & Within population & 31 & $709,215.400$ & 2877.900 & 24 \\
\hline & Total & 38 & $1,187,165.500$ & $12,197.000$ & \\
\hline \multirow[t]{4}{*}{ (D) } & L. glauca & & & & \\
\hline & Among population & 28 & 1005.172 & 2.706 & 9 \\
\hline & Within population & 76 & 2007.043 & 26.066 & 91 \\
\hline & Total & 104 & 3012.215 & 28.772 & \\
\hline
\end{tabular}

Pairwise Mantel tests for L. praecox (Figure 2a), $F_{\mathrm{ST}}$ and distance were positively correlated ( $p=0.025$ ). For L. umbellata (Figure $2 b$ ) indicated no statistically significant correlation between $F_{\mathrm{ST}}$ and the geographic distance between populations $(p=0.897)$. Lindera obtusiloba and L. glauca (Figure 2c,d) also showed $F_{\mathrm{ST}}$ and distance were positively correlated ( $p=0.023$ and 0.010 , respectively).

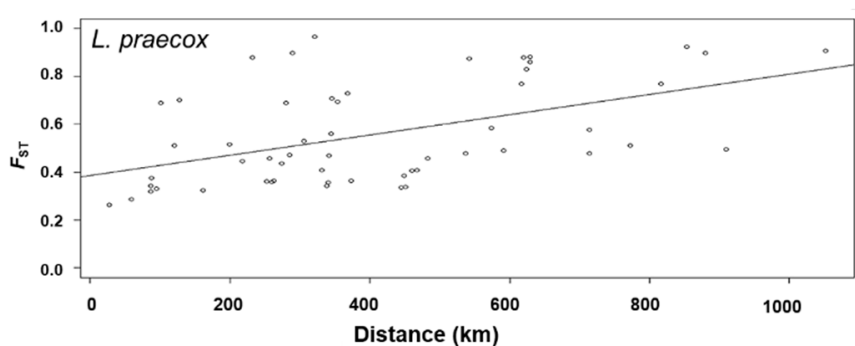

(a)

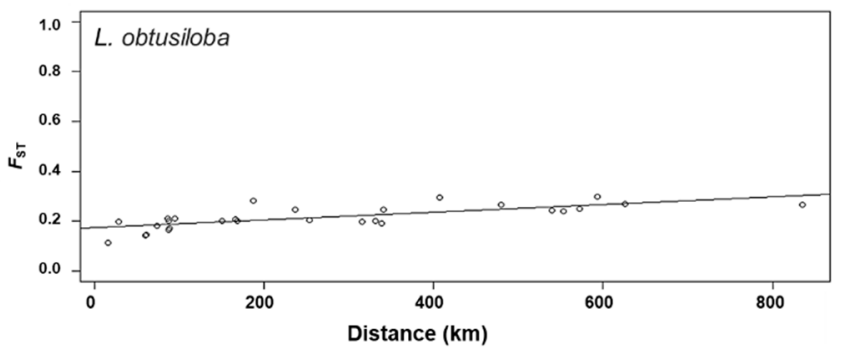

(c)

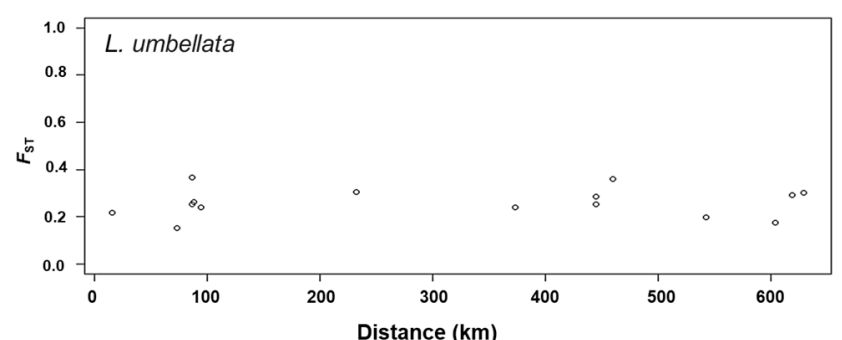

(b)

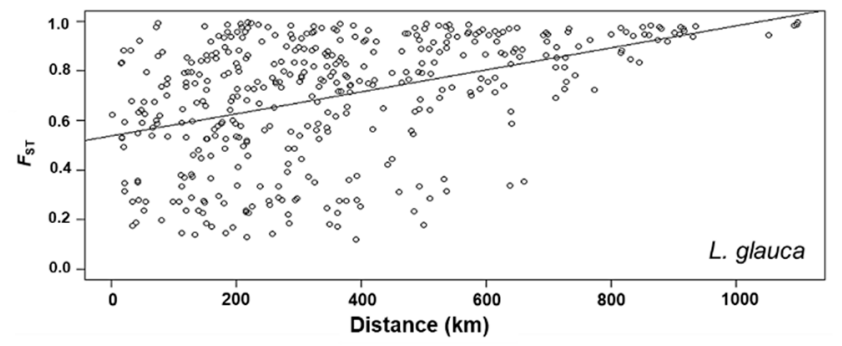

(d)

Figure 2. Mantel test for the correlation between $F_{\mathrm{ST}}$ and geographic distance among populations of (a) L. praecox ( $\left.p=0.025\right)$, (b) L. umbellata ( $p=0.897)$, (c) L. obtusiloba $(p=0.023)$, and (d) L. glauca $(p=0.010)$.

The PCoA of L. praecox and L. obtusiloba appears to have genetically distinct variation within their range from east to west (Figure 3a,c), and geographic genetic differentiation of L. umbellata was clear but its pattern was complex (Figure 3b). The PCoA plot of L. glauca showed no clear differentiation in genetic composition throughout its geographic range in Japan (Figure 3d). 

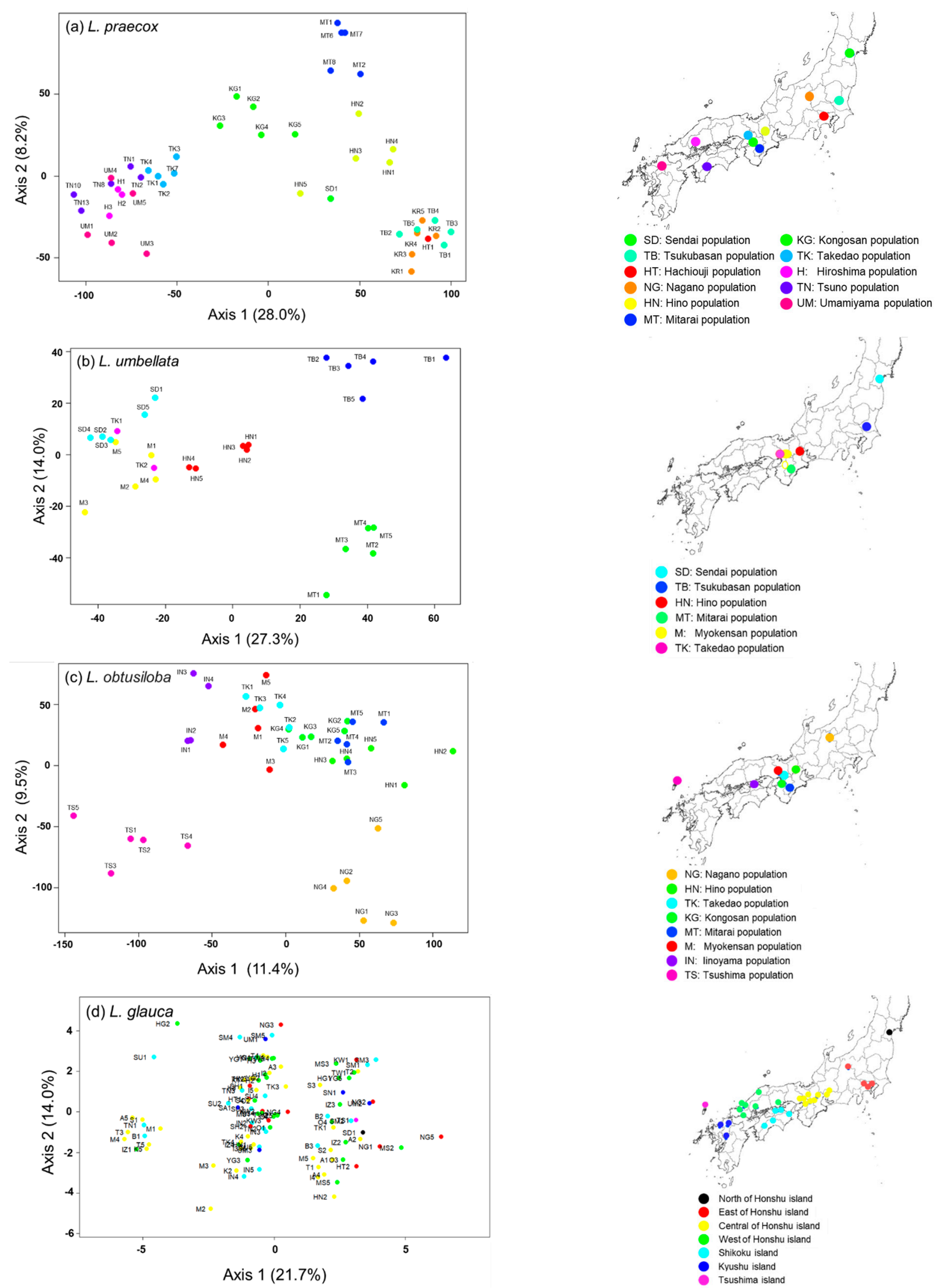

Figure 3. Principal coordinate analysis (PCoA) plot of (a) 45 individuals of L. praecox, (b) 27 individuals of $L$. umbellata, (c) 39 individuals of L. obtusiloba, and (d) 105 individuals of L. glauca. Percentages of total variation explained by each coordinate are shown in parentheses. The marks on the map indicate the location of each population. 


\section{Discussion}

A key advantage of MIG-seq is that it can provide putative neutral loci [28], which are useful for analyses of genetic structure. Therefore, this approach has been used recently in population genetics (e.g., $[29,30])$.

Obligate outcrossing species is expected to have the values of Ho close to those of He as reported by Ye et al. [31] for L. obtusiloba. For the genetic diversity of the dioecious tree species Phoebe zhennan (Lauraceae), Ho was higher than $\mathrm{He}(\mathrm{Ho}=0.243, \mathrm{He}=0.145)$, indicating low inbreeding [32]. Our results are similar to those of previous studies. This may be an advantage of dioecy, which avoids inbreeding. Our study results also suggest that dioecious plants have greater heterozygosity than cosexual plants, based on a comparison

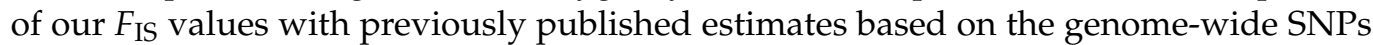
data (Tables 2-4), 0.035-0.091 (Rhododendron japonoheptamerum) [29]; 0.05-0.19 (Platycladus orientalis) [33]; 0.05 (Thuja koraiensis) [33]; 0.051-0.754 (Tectona grandis) [30]). However, the level of heterozygosity is influenced not only by reproductive system of the focal species, but also other factors, e.g., population size [34], age of individuals [35]. Thus, careful verification is needed.

The all-female populations of L. glauca assessed here showed higher heterozygosity than the other three Lindera species, on average, and exhibited negative $F_{\text {IS }}$ values at all sites except for one site. These results are similar to a previous study that employed nuclear microsatellite analyses [18]. Zhu et al. [18] suggested that seven Japanese populations of L. glauca exhibited greater heterozygosity than what was observed across a large range of populations in China. For an apomictic lineage, the high and well-preserved heterozygosity in L. glauca populations in Japan may attribute to asexual (apomictic) reproduction as reported for Prunus avium [36], due to the absence of sexual recombination and/or the lack of accumulation of somatic mutations over time.

We found evidence of genetic differentiation among populations of L. praecox, L. umbellata, and L. obtusiloba as has often been reported for other plant species [37-39]. First, AMOVA results indicated that regional variation explained a large proportion of the total variation (Table 5). Second, the PCoA results indicated geographic divisions in populations. Finally, we found that populations that were further apart in space were more genetically different in L. praecox and L. obtusiloba (Figure 2a,c). Collectively, these results reflect geographically limited gene flow, a varied history of population expansion, or strong adaptation to local environmental conditions.

By contrast, populations of L. glauca had substantially lower genetic variation than these three species. Even in apomictic plants, genetic diversity can occur in the process of seed production by incomplete meiosis and/or recombination [40]. In the case of L. glauca in Japan, the comparison of multi-level genetic distance and PCR error rates indicated no significant differences for any level, which suggests that seeds were exact copies of their mothers, as the genetic difference between mothers and seeds were within the range of PCR errors (Figure 1d). A seed production process that does not produce mutations would lead genetically uniform populations in Japan.

Here, we compare our own results in Japan with available results from China. Lindera obtusiloba produces seeds by sexual mating both in China and Japan. Genetically distinct populations in different regions were observed in China [31]. Our results are similar to those of the previous study. On the other hand, the situation is different for L. glauca. Both females and males of Lindera glauca exist in China, and they reproduce sexually. A variety of genotypes existed in different regions and geographic genetic structure was observed in China [18]. While in Japan, seed production by apomixis resulted in an extremely uniform genetic structure. These results of comparisons suggest that reproductive system has a strong influence on genetic structure of plant populations.

We were surprised that virtually no genetic variation was observed in L. glauca across its entire geographic range in Japan. The genetic difference between individuals in the whole range of Japan was not significantly different from PCR errors (Figure 1d). In addition, we found significant but weak correlation between genetic and geographic 
distance (Figure 2d), and the PCoA results indicated unclear geographic distinctions among populations (Figure 3d). These are contrast to the previous studies which detected a clear geographic genetic structure of apomictic plants, Miconia albicans [7] and Taraxacum officinale [9]. These results suggested that a population of L. glauca in Japan might be a huge clone. Bricker et al. [41] reported that a dioecious aquatic plant, Thalassia testudinum, formed a "mega-clone" that spread over nearly $50 \mathrm{~km}$ by vegetative fragments. The European populations of Gagea spathacea (Liliaceae) were dominated by a single "megaclone" spreading all over Central Europe [42]. Similarly, the L. glauca population in Japan could also be considered as a "mega-clone" that spread out over at least $1100 \mathrm{~km}$ in distance from Sendai to Kumamoto. There are two possible reasons for the formation of genetically uniform populations. First, Japanese populations of L. glauca might be founded by one asexually reproducing female as suggested by Dupont [17]. Second, population of L. glauca in Japan may still be young on the geological time scale. The population of L. glauca has been in Japan since prehistoric times because Pleistocene fossils of L. glauca have been found [11]. Genetic diversification via accumulated mutations has not yet occurred, although it may occur in the future. However, there were significant dissimilarities in the degree of the genetic difference between a mother plant and its seeds, between individuals in a population, and between individuals in the entire study area (Figure 1d). The result suggested that slight mutations may have occurred and accumulated.

Although L. glauca has been present in Japan since the Pleistocene (0.01-2.58 million years ago (Ma)) [11], the genetic diversity of this species has remained at a fairly low level until now. According to Xiong et al. [19], wild L. glauca in mainland China have a highly skewed sex ratio with females predominating, and their genetic diversity is thought to be maintained by sexual reproduction with a small number of male plants. It is not clear whether Japanese L. glauca can cross with the Chinese males. However, if they can, immigration of males may increase genetic diversity as well as the Chinese L. glauca.

Finally, the loss of genetic diversity relates to an increase in extinction risk [43]. Despite this, populations with extremely low genetic diversity have been reported worldwide [42,44]. In the apomictic L. glauca, the advantages of population establishment or expansion by a single individual, without mating, may outweigh the disadvantages associated with the extremely low genetic diversity. If environmental conditions remain suitable for the extant genotype, genetic diversity may not be required to maintain these populations, at least in the short term.

\section{Conclusions}

The three species that produce seeds by sexual reproduction, Lindera praecox, L. umbellata, and L. obtusiloba had lower values for inbreeding coefficient, indicating that there is indeed an effect of dioecy. Genetic variations were also found both at the local scale and the geographic scale. It is possible that they have higher genetic diversity than cosexual plants, and the effect of inbreeding depression may be suppressed. On the other hand, in L. glauca, the advantage of being able to found populations from single individual, without mating, may outweigh the disadvantages associated with extremely low genetic diversity. This may explain why this species is so widely distributed in Japan. Provided that the current genotypes remain suited to environmental conditions, L. glauca may not be constrained by its limited genetic diversity.

Supplementary Materials: The following are available online at https:/ / www.mdpi.com/1999-490 7/12/2/227/s1, Table S1; sampling locations and sample size of leaf collection of the four Lindera species, Table S2; sampling locations and sample size of seeds of the four Lindera species.

Author Contributions: Conceptualization, M.N. and S.N.; formal analysis, M.N., S.N., and S.O.; investigation, M.N., S.N., S.O., S.K.H., A.M., Y.S., H.T., and S.Y.; writing-original draft preparation, M.N., S.N., and A.I.; funding acquisition, S.N. and A.I. All authors have read and agreed to the published version of the manuscript.

Funding: This study was supported by KAKENHI grants (16K07783, 18H02224) from the Japan Society for the Promotion of Science, Japan. 
Acknowledgments: We thank the Nara Park Management Office for permission to work in the Kasugayama Forest Reserve, and Itsuki Ohya, Saki Takeda, Mayu Tatsumi, and Muneto Hirobe for their assistance in the field work. We also thank Junko Muramatsu for assistance in the experiments. We wish to thank Yuji Isagi for his helpful comments.

Conflicts of Interest: The authors declare no conflict of interest.

Accession Codes: Raw MIG-seq data are deposited at the DDBJ Sequence Read Archive (DRA) with accession numbers DRA011268 (Submission), PRJDB10877 (BioProject), SAMD00262038-SAMD00262 042 and SAMD00261073-SAMD00261218 (BioSample), DRX250152-DRX250529 (Experiment) and DRR260449-DRR 260826 (Run).

\section{References}

1. Booy, G.; Hendriks, R.J.J.; Smulders, M.J.M.; Van Groenendael, J.M.; Vosman, B. Genetic diversity and the survival of populations. Plant Biol. 2000, 2, 379-395. [CrossRef]

2. Booth, R.E.; Grime, J.P. Effects of genetic impoverishment on plant community diversity. J. Ecol. 2003, 91, 721-730. [CrossRef]

3. Hamrick, J.L.; Godt, M.J.W. Effects of life history traits on genetic diversity in plant species. Philos. Trans. R. Soc. Lond. Ser. B Biol. Sci. 1996, 351, 1291-1298. [CrossRef]

4. Muyle, A.; Martin, H.; Zemp, N.; Mollion, M.; Gallina, S.; Tavares, R.; Silva, A.; Bataillon, T.; Widmer, A.; Glémin, S.; et al. Dioecy is associated with high genetic diversity and adaptation rates in the plant genus Silene. Mol. Biol. Evol. 2020. [CrossRef] [PubMed]

5. Nanami, S.; Kawaguchi, H.; Yamakura, T. Dioecy-induced spatial patterns of two codominant tree species, Podocarpus nagi and Neolitsea aciculata. J. Ecol. 1999, 87, 678-687. [CrossRef]

6. Lo, E.Y.Y.; Stefanović, S.; Dickinson, T.A. Population genetic structure of diploid sexual and polyploid apomictic hawthorns (Crataegus; Rosaceae) in the Pacific Northwest. Mol. Ecol. 2009, 18, 1145-1160. [CrossRef]

7. Dias, A.C.C.; Serra, A.C.; Sampaio, D.S.; Borba, E.L.; Bonetti, A.M.; Oliveira, P.E. Unexpectedly high genetic diversity and divergence among populations of the apomictic Neotropical tree Miconia albicans. Plant Biol. 2018, 20, 244-251. [CrossRef] [PubMed]

8. Tokumoto, Y.; Matsushita, M.; Kishimoto-Yamada, K.; Nikkeshi, A.; Isogimi, T.; Nakagawa, M. Floral visitors and reproductive success in two sequentially flowering Lindera shrubs (Lauraceae) of central Japan. J. For. Res. 2019, 24, 42-51. [CrossRef]

9. Majeský, L'.; Vašut, R.J.; Kitner, M.; Trávníček, B. The pattern of genetic variability in apomictic clones of Taraxacum officinale indicates the alternation of asexual and sexual histories of apomicts. PLoS ONE 2012, 7, 41868. [CrossRef]

10. Onoe, T. Palaeoenvironmental analysis based on the Pleistocene Shiobata flora in the Shiobata volcanic basin, central Japan. Rep. Geol. Surv. Jpn. 1989, 269, 1-207, (in Japanese with English Summary).

11. Onoe, T. A Pleistocene flora from Ebino City, Miyazaki Prefecture, Japan. Rep. Geol. Surv. Jpn. 1971, 241, 1-46, (in Japanese with English Summary).

12. Dupont, Y.L.; Kato, M. Sex ratio variation in dioecious plant species: A comparative ecological study of six species of Lindera (Lauraceae). Nord. J. Bot. 1999, 19, 529-540. [CrossRef]

13. Watanabe, M. Encyclopedia of Forestry; Japan Forest Technology Association: Maruzen, Tokyo, Japan, 1961; p. 11. (In Japanese)

14. Yagi, M.; Takabe, W.; Matsumi, S.; Shimode, A.; Maruyama, T.; Yonei, Y. Screening and selection of anti-glycative materials: Kuromoji (Lindera umbellata). Glycative Stress Res. 2017, 4, 317-328. [CrossRef]

15. Choi, H.G.; Choi, Y.H.; Kim, J.H.; Kim, H.-H.; Kim, S.-H.; Kim, J.A.; Lee, S.M.; Na, M.; Lee, S.H. A new neolignan and lignans from the stems of Lindera obtusiloba Blume and their anti-allergic inflammatory effects. Arch. Pharm. Res. 2014, 37, 467-472. [CrossRef] [PubMed]

16. Niu, J.; Chen, Y.; An, J.; Hou, X.; Cai, J.; Wang, J.; Zhang, Z.; Lin, S. Integrated transcriptome sequencing and dynamic analysis reveal carbon source partitioning between terpenoid and oil accumulation in developing Lindera glauca fruits. Sci. Rep. UK 2017, 5, 15017. [CrossRef]

17. Dupont, Y.L. Evolution of apomixis as a strategy of colonization in the dioecious species Lindera glauca (Lauraceae). Popul. Ecol. 2002, 44, 293-297. [CrossRef]

18. Zhu, S.S.; Comes, H.P.; Tamaki, I.; Cao, Y.N.; Sakaguchi, S.; Yap, Z.Y.; Ding, Y.Q.; Qiu, Y.X. Patterns of genotype variation and demographic history in Lindera glauca (Lauraceae), an apomict-containing dioecious forest tree. J. Biogeogr. 2020, 47, 2002-2016. [CrossRef]

19. Xiong, B.; Zhang, L.; Dong, S.; Zhang, Z. Population genetic structure and variability in Lindera glauca (Lauraceae) indicates low levels of genetic diversity and skewed sex ratios in natural populations in mainland China. PeerJ 2020, 8, 8304. [CrossRef]

20. Suyama, Y.; Matsuki, Y. MIG-seq: An effective PCR-based method for genome-wide single-nucleotide polymorphism genotyping using the next-generation sequencing platform. Sci. Rep. UK 2015, 5, 16963. [CrossRef]

21. Catchen, J.M.; Amores, A.; Hohenlohe, P.; Cresko, W.; Postlethwait, J.H. Stacks: Building and genotyping loci de novo from short-read sequences. G3-Genes Genom. Genet. 2011, 1, 171-182. [CrossRef]

22. Catchen, J.; Hohenlohe, P.A.; Bassham, S.; Amores, A.; Cresko, W.A. Stacks: An analysis tool set for population genomics. Mol. Ecol. 2013, 22, 3124-3140. [CrossRef] 
23. Peakall, R.; Smouse, P.E. GenAlEx 6.5: Genetic analysis in Excel. Population genetic software for teaching and research-an update. Bioinformatics 2012, 28, 2537-2539. [CrossRef]

24. Smouse, P.E.; Peakall, R. Spatial autocorrelation analysis of individual multiallele and multilocus genetic structure. Heredity 1999, 82, 561-573. [CrossRef]

25. Paradis, E. pegas: An R package for population genetics with an integrated-modular approach. Bioinformatics 2010, 26, 419-420. [CrossRef]

26. Oksanen, J.F.; Guillaume, B.; Friendly, M.; Kindt, R.; Legendre, P.; McGlinn, D.; Minchin, P.R.; O’hara, R.B.; Simpson, G.L.; Solymos, P.; et al. Vegan: R package version. Community Ecol. Package 2019, 2, 1-259.

27. Paradis, E.; Schliep, K. ape 5.0: An environment for modern phylogenetics and evolutionary analyses in R. Bioinformatics 2019, 35, 526-528. [CrossRef]

28. Richards, Z.T.; Yasuda, N.; Kikuchi, T.; Foster, T.; Mitsuyuki, C.; Stat, M.; Suyama, Y.; Wilson, N.G. Integrated evidence reveals a new species in the ancient blue coral genus Heliopora (Octocorallia). Sci. Rep. Orts. 2018, 8, 15875. [CrossRef]

29. Tamaki, I.; Yoichi, W.; Matsuki, Y.; Suyama, Y.; Mizuno, M. Inconsistency between morphological traits and ancestry of individuals in the hybrid zone between two Rhododendron japonoheptamerum varieties revealed by a genotyping-by-sequencing approach. Tree Genet. Genomes 2017, 13, 1-10. [CrossRef]

30. Prasetyo, E.; Widiyatno; Indrioko, S.; Na'iem, M.; Matsui, T.; Matsuo, A.; Suyama, Y.; Tsumura, Y. Genetic diversity and the origin of commercial plantation of Indonesian teak on Java Island. Tree Genet. Genomes 2020, 16, 1-14. [CrossRef]

31. Ye, J.W.; Bai, W.N.; Bao, L.; Wang, T.M.; Wang, H.-F.; Ge, J.P. Sharp genetic discontinuity in the aridity-sensitive Lindera obtusiloba (Lauraceae): Solid evidence supporting the Tertiary floral subdivision in East Asia. J. Biogeogr. 2017, 44, 2082-2095. [CrossRef]

32. Xiao, J.-H.; Ding, X.; Li, L.; Ma, H.; Ci, X.-Q.; van der Merwe, M.; Conran, J.G.; Li, J. Miocene diversification of a golden-thread nanmu tree species (Phoebe zhennan, Lauraceae) around the Sichuan Basin shaped by the East Asian monsoon. Ecol. Evol. 2020, 10, 10543-10557. [CrossRef] [PubMed]

33. Jia, K.; Zhao, W.; Maier, P.A.; Hu, X.; Jin, Y.; Zhou, S.; Jiao, S.; El-Kassaby, Y.A.; Wang, T.; Wang, X.; et al. Landscape genomics predicts climate change-related genetic offset for the widespread Platycladus orientalis (Cupressaceae). Evol. Appl. 2020, 13, 665-676. [CrossRef]

34. González-Martínez, S.C.; Krutovsky, K.V.; Neale, D.B. Forest-tree population genomics and adaptive evolution. New Phytol. 2006, 170, 227-238. [CrossRef]

35. Belletti, P.; Monteleone, I.; Ferrazzini, D. Genetic variability at allozyme markers in sycamore (Acer pseudoplatanus) populations from northwestern Italy. Can. J. For. Res. 2007, 37, 395-403. [CrossRef]

36. Stoeckel, S.; Grange, J.; Fernández-Manjarres, J.F.; Bilger, I.; Frascaria-Lacoste, N.; Mariette, S. Heterozygote excess in a selfincompatible and partially clonal forest tree species-Prunus avium L. Mol. Ecol. 2006, 15, 2109-2118. [CrossRef]

37. Stojnić, S.; Avramidou, E.; Fussi, B.; Westergren, M.; Orlović, S.; Matović, B.; Trudić, B.; Kraigher, H.; Aravanopoulos, F.; Konnert, M. Assessment of genetic diversity and population genetic structure of Norway spruce (Picea abies (L.) Karsten) at Its Southern lineage in Europe. Implications for conservation of forest genetic resources. Forests 2019, 10, 258. [CrossRef]

38. Al-Qthanin, R.N.; Alharbi, S.A. Spatial structure and genetic variation of a mangrove species (Avicennia marina (Forssk.) Vierh) in the Farasan Archipelago. Forests 2020, 11, 1287. [CrossRef]

39. Cai, M.; Wen, Y.; Uchiyama, K.; Onuma, Y.; Tsumura, Y. Population genetic diversity and structure of ancient tree populations of Cryptomeria japonica var. sinensis based on RAD-seq data. Forests 2020, 11, 1192. [CrossRef]

40. Van Baarlen, P.; van Dijk, P.J.; Hoekstra, R.F.; de Jong, J.H. Meiotic recombination in sexual diploid and apomictic triploid dandelions (Taraxacum officinale). Stadler Gen. 2000, 43, 827-835.

41. Bricker, E.; Calladine, A.; Virnstein, R.; Waycott, M. Mega clonality in an aquatic plant-a potential survival strategy in a changing environment. Front. Plant Sci. 2018, 9, 435. [CrossRef] [PubMed]

42. Pfeiffer, T.; Klahr, A.; Peterson, A.; Levichev, I.G.; Schnittler, M. No sex at all? Extremely low genetic diversity in Gagea spathacea (Liliaceae) across Europe. Flora Morphol. Distrib. Funct. Ecol. Plants 2012, 207, 372-378. [CrossRef]

43. Frankham, R. Genetics and extinction. Biol. Conserv. 2005, 126, 131-140. [CrossRef]

44. Rasmussen, K.K.; Kollmann, J. Low genetic diversity in small peripheral populations of a rare European tree (Sorbus torminalis) dominated by clonal reproduction. Conserv. Genet. 2008, 9, 1533-1539. [CrossRef] 